\title{
Inching Locomotion for Planetary Rover Mobility
}

\author{
Scott Moreland, Krzysztof Skonieczny, David Wettergreen \\ Robotics Institute, Carnegie Mellon University \\ 5000 Forbes Ave \\ Pittsburgh, PA 15213 \\ smoreland@cmu.edu \\ Vivake Asnani, Colin Creager, Heather Oravec \\ Tribology \& Mechanical Systems Branch, NASA Glenn Research Center \\ 21000 Brookpark Road \\ Cleveland, $\mathrm{OH} 44135$
}

\begin{abstract}
New articulated planetary rovers offer alternative locomotion modalities beyond conventional rolling wheel mobility. ${ }^{12}$ These new modalities should be explored to overcome the limitations of traditional rolling mobility, and expand the areas of planetary surfaces amenable to exploration. The topic of this study is a hybrid push-roll locomotion mode called inching. Static (non-rolling) wheels are used in conjunction with the rolling wheels of a vehicle in order to increase net traction potential. Preliminary experiments have shown an approximate doubling in drawbar pull for the inching locomotion mode relative to pure rolling. This improvement is not accounted for by reductions to wheel motion resistance alone, and furthermore evidence is provided that static wheels are capable of reacting more ground thrust than rolling wheels. Further investigations using a transparent soil tank, and novel image processing techniques, reveal key differences in the soil shear failure interface under rolling and static wheels. For the cases studied, static wheels generated much deeper and more unified soil failure masses than rolling wheels. Further investigation is recommended to clarify the physics of these thrust development processes, and ultimately to populate the vehicle design space for inching locomotion.
\end{abstract}

\section{TABLE OF ConTents}

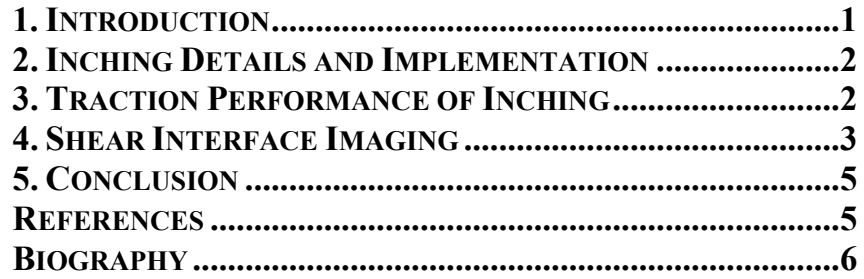

\section{INTRODUCTION}

Vehicles with a variety of articulation types are being developed for exploration of the Moon and other planetary bodies [1 - 4]. This has allowed for modes of locomotion and methods of operation unlike previously deployed planetary rovers. Systems that are capable of conventional rolling, walking, hybrid push-roll and center of gravity

\footnotetext{
${ }^{1}$ 978-1-4244-7351-9/11/\$26.00 C2011 IEEE

${ }^{2}$ IEEEAC paper \#1803, Version 11, Updated January 14, 2011
}

control have been developed and are being considered for missions.

Inching is a hybrid locomotion mode, where the vehicle wheel base is expanded and contracted using onboard actuation. By this action, a set of rolling wheels can be assisted by pushing or pulling off of a set of non-rolling wheels. Net traction gains have been observed, which have been attributed to either a decrease in motion resistance or an increase thrust available from the non-rotating set of wheels. Therefore in inchworm mode, a vehicle could potentially climb higher slopes or tow a greater payload than in rolling mode. The vehicle could also lower its slip rate and/or escape from an entrenched state in loose terrain. The analysis of inching locomotion has been previously undertaken for both planetary exploration and military vehicles [5 - 8]. However, these studies have not sufficiently investigated the different traction phenomena of both the rolling and inching wheel.

A study of the European Space Agency (ESA) ExoMars rover system provides the latest example of a mobility system that benefits from inching like locomotion $[3,13]$. Similarly, the JPL ATHLETE system and the NASA Space Exploration Vehicle (SEV), are highly articulated, but there isn't enough information to determine how to best take advantage of the inching technique.

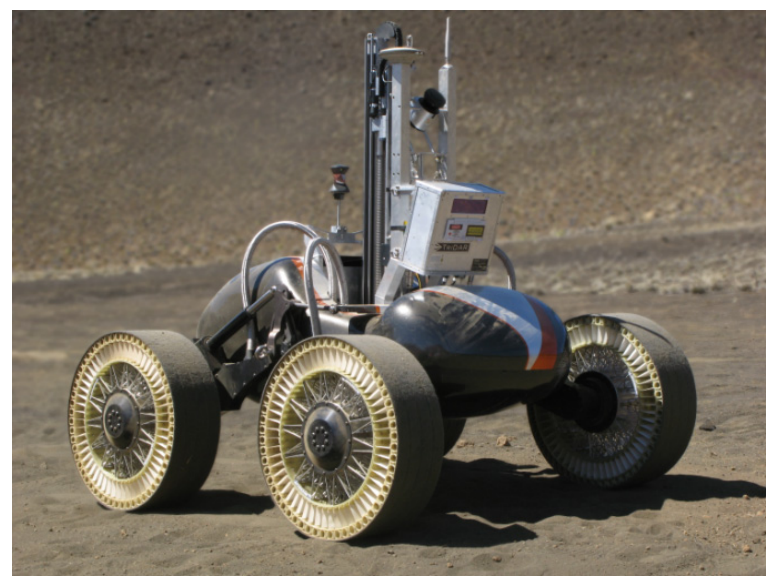

Figure 1. The Scarab rover has an actively controlled suspension that can change wheel base length. 


\section{INCHING DETAILS AND IMPLEMENTATION}

Inching is a specific implementation of the hybrid push-roll locomotion technique, which is intended to increase the net traction of a vehicle. Net traction is defined as the groundthrust force that is available to do work, above what is needed to simply overcome rolling resistance. By rolling two out of four wheels, for example, motion resistance is cut in half. Additionally, inching mode may increase the available thrust if the wheel-terrain system can support more thrust in a static mode rather than during rolling.

The inching mode of operation can be achieved by the Scarab rover (Fig. 1); a concept vehicle developed at Carnegie Mellon University under the NASA Exploration Technology Development Program. This system has demonstrated, on a full scale prototype, inching locomotion in laboratory and in field settings that are analogous to lunar terrain [4].

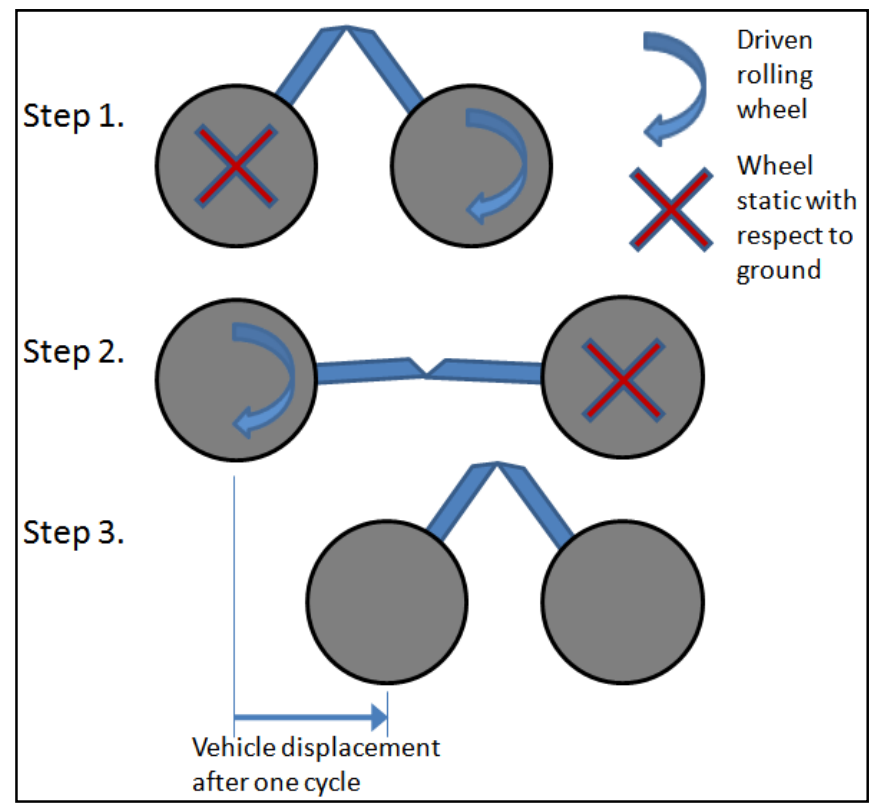

Figure 2. Simplified kinematics of the Scarab suspension demonstrating inching, with indication of the suspension and wheel motions.

To begin the cycle of inching on Scarab (Fig. 2), the wheelbase expands while rolling the front wheels forward and keeping the rear wheels fixed relative to the ground. Once the limit of body expansion is reached, the wheel base contracts while the rear wheels roll forward and the front wheels remain fixed with respect to the ground. Note that, the fixed wheel actuators must counter-rotate in synchrony with the expanding or contracting side frame; if they were to be locked they would rotate in the direction of vehicle displacement and thereby inducing slippage associated with a rotating wheel. These non-rotating wheels can provide extremely high thrust. To achieve these benefits, inching locomotion not only eliminates the wheel motion resistances on two of the four wheels by remaining fixed with respect to ground, but it also builds high strength in the soil to provide increased thrust.

\section{TRACTION PERformanCE OF INCHING}

Laboratory drawbar pull testing [9] of Scarab was done to compare the tractive capabilities of the rolling and inching modes of locomotion. In this test, a "drawbar load" is applied to the vehicle as an external resistance to driving. Thus, the applied drawbar load is equivalent to the net traction potential (thrust available in excess of wheel motion resistance) of the vehicle for the specific environmental and driving conditions. During the test, the vehicle tows the drawbar load with predetermined wheel speed. In the case of inching mode, the wheel speed and rate of wheel base expansion are predetermined. The wheel rotational displacement and vehicle translational displacement are measured, from which the wheel slip is determined. The metric slip or slip ratio as utilized in this study can more specifically be defined as travel reduction. Travel reduction is the percentage the vehicle ground speed is reduced by due to combined wheel slippage. A near zero load case in which only self propelled motion resistances are present is utilized as the baseline speed assumed to be zero slip (although it is non-zero but sufficiently low). The measured self propelled ground speed of this case is used in all subsequent inching slip metrics under varying drawbar loading. The slip metric formula is as follows:

$$
\text { Slip Ratio }=1-\frac{\text { drawbar pull test ground speed }}{\text { self propelled ground speed }}
$$

In order to create a drawbar pull curve, a range of loads must be applied in steps that span $0 \%$ to $100 \%$ slip at steady state response. The drawbar pull value is quite informative when comparing different aspects of wheel and suspension designs during physical testing but can also be used for estimating the slope a vehicle can continuously ascend under low slip for a specified material. Drawbar pull is usually expressed as pull load normalized by vehicle weight [7].

Drawbar pull tests were conducted at the NASA Glenn Research Center's Simulated Lunar OPErations laboratory (SLOPE). A cable payout mechanism with variable tension control (via a magnetic brake) was used to apply the drawbar load to the vehicle (Fig. 3). Wheel rotational displacement was monitored using encoders and vehicle translational displacement was determined using a 3-axis laser tracking system.

The vehicle was configured at $400 \mathrm{~kg}$ mass, with $\varnothing 71 \mathrm{~cm} \mathrm{x}$ $25 \mathrm{~cm}$ wide rigid wheels and driven on GRC-1 lunar soil strength simulant. This soil was prepared before each test to achieve terrain strength and deformation properties similar to that found on the lunar surface [10]. Prior to each drawbar pull test, the soil was fully loosened (to $45 \mathrm{~cm}$ depth) and then compacted with a heavily loaded roller. Cone penetration resistance measurements before each 
drawbar pull test were used to check that the specific pressure-density gradient was achieved.

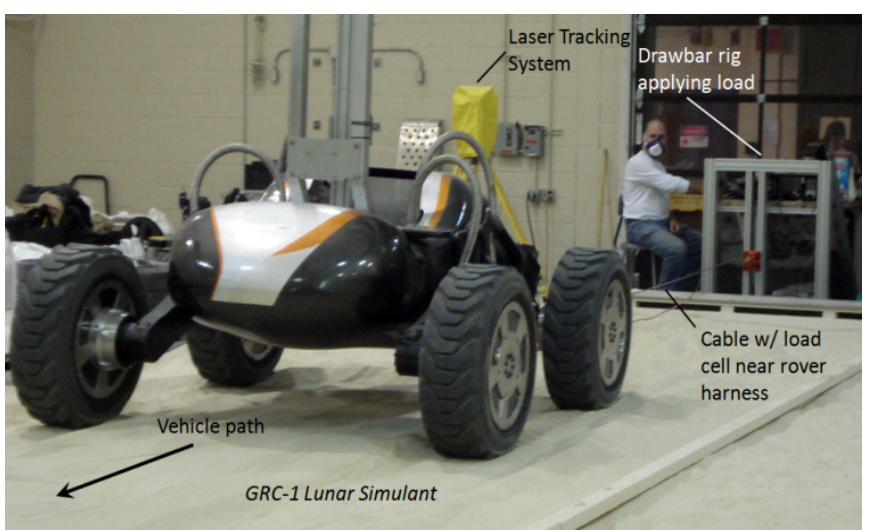

Figure 3. Scarab rover undergoing drawbar pull test to evaluate vehicle tractive performance. The Drawbar Rig applied a constant controlled load via a magnetic brake while a laser ranging system measured vehicle location.

Several load conditions were tested, and load (normalized by the vehicle weight) vs. slip was plotted for both inching and conventional rolling (Fig. 4). The results showed that for the entire range of slip, inching produces approximately $100 \%$ more drawbar pull than rolling. For example, at a slip ratio of about 0.3 , the rolling drawbar pull is $11 \%$, while the inching drawbar pull is $21 \%$.

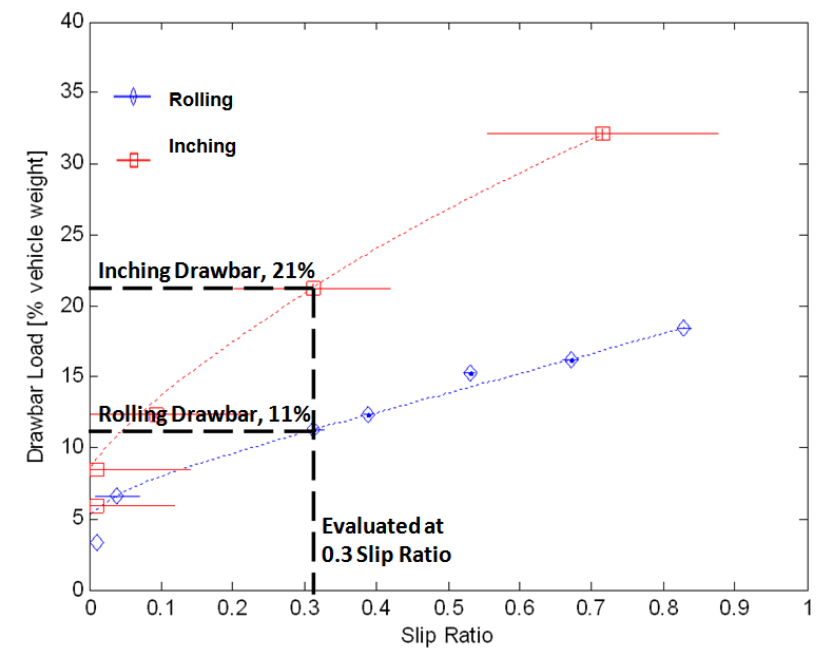

Figure 4. Drawbar load vs. slip comparing rolling and inching modes with other parameters (mass, wheels, soil condition) held constant. Results indicate an approximate doubling of drawbar pull.

Increases in net thrust of this magnitude cannot be explained solely by reductions in wheel motion resistance, and therefore it is hypothesized that there is a significant difference in the thrust potential between rolling and static wheels. This observation motivated a comparative study into the traction mechanics. An experiment was therefore designed to image the soil motion beneath wheels during rolling and static modes of generating thrust.

\section{SHEAR INTERFACE IMAGING}

An experimental apparatus (Fig. 5) was designed to image the shear interface of the soil (Fig. 6). The rig consisted of a glass walled soil bin that could move horizontally, and a wheel that could move free vertically. Dead weight was used to apply a vertical payload to the wheel, a pulley system was used to pull the soil tank and create a constant horizontal wheel load. During rolling mode experiments, a motor was used to rotate the wheel at a constant rate. During static experiments, the motor was used to brake the wheel and keep it from rotating. Images of the wheel rim and soil were taken using a high speed camera. These images were analyzed by means of an optical flow/clustering algorithm to show soil shear failure planes and soil particle velocities.

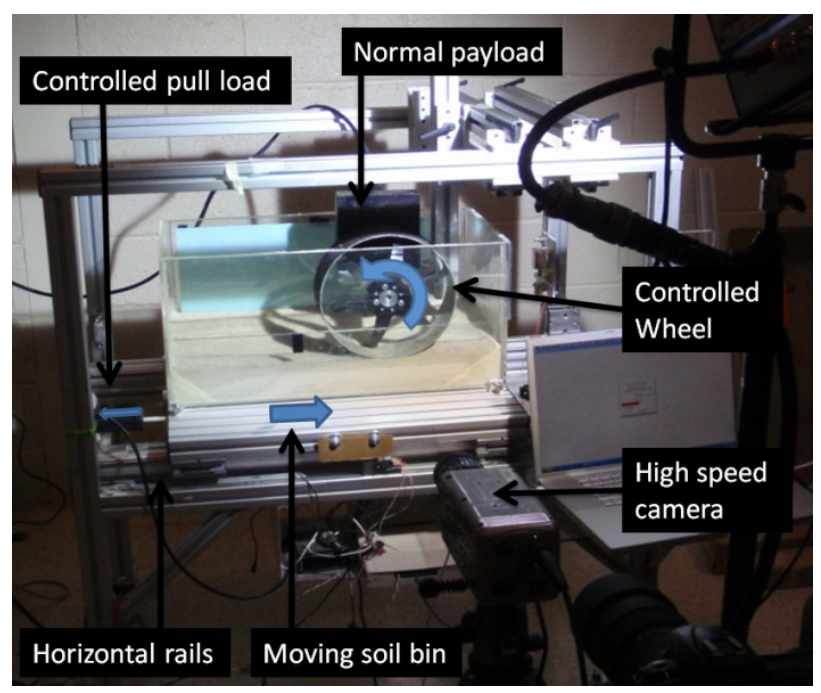

Figure 5. Shear interface imaging rig consisted of a moving transparent soil bin on horizontal rails with a controlled drawbar pull load applied to a rolling or static wheel. The subsurface soil motion is imaged using a high speed camera.

The normal load condition for both the rolling and inching wheels was $13.5 \mathrm{~kg}$, while the horizontal pull load was $2 \mathrm{~kg}$ and $6 \mathrm{~kg}$ respectively $(0.15$ and 0.44 drawbar normalized by normal load). The payload value was chosen based on typical ground pressure requirements for planetary surface systems $(69-83 \mathrm{kPa}$ average) to achieve low sinkage and adequate traction. The ground pressure value was estimated by measurement of the contact area during steady state operation of the rolling wheel and this payload was applied to both rolling and static wheels. The wheel dimensions were $\varnothing 24.5 \mathrm{~cm} \times 18.5 \mathrm{~cm}$ wide with a rigid rim and sandpaper like traction surface. The pull load was chosen to be slightly above the failure load for both locomotion cases. Although these higher than nominal loads are not the exact conditions a vehicle would typically operate at, these loads should approximate the maximum possible thrusts. For the rolling wheel, a high slip case, near failure, was chosen (about 0.5 slip ratio), while the non-rotating wheel was 
given load slightly above full soil failure (infinite negative slip). The actual load values were determined experimentally by stepping up from zero load until failure.
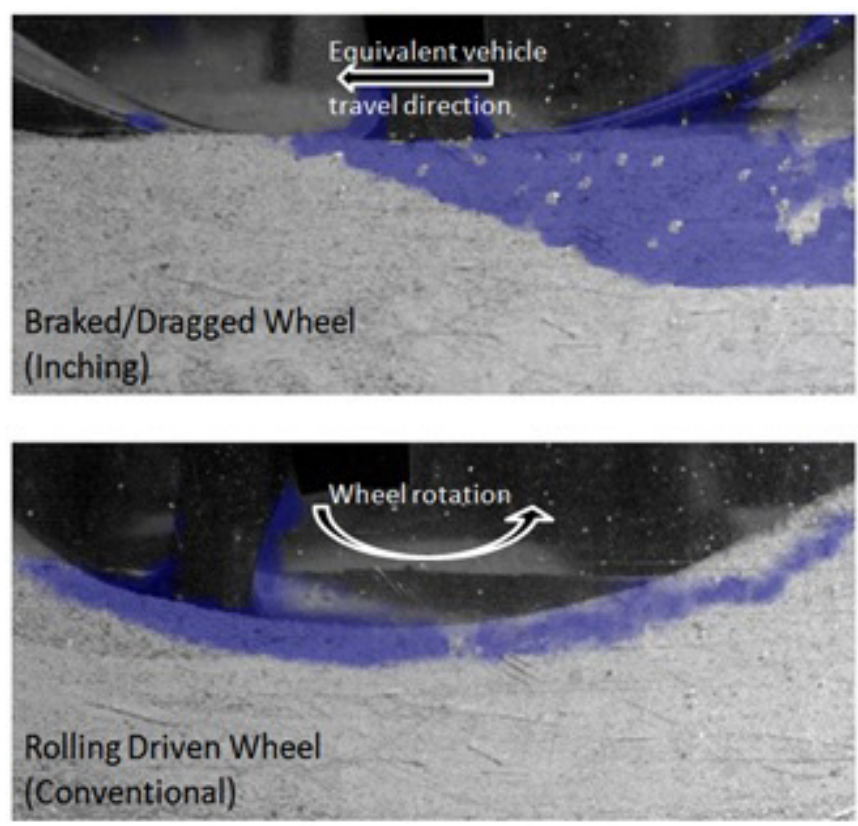

Figure 6. Processed high-speed video of rolling and inching experiments displaying shear interface for both locomotion types. Blue/dark regions show material within the sheared soil mass. The boundary between blue/dark and light color indicate the soil failure planes. The inching wheel was analyzed at the onset of loading.

The image processing implemented relies on optical flow and clustering techniques. The optical flow algorithm [11] tracks displacement of soil regions relative to a prior frame and calculates a motion vector for each pixel. The blue colored regions of Fig. 6 show regions clustered as 'moving' sand (within the shear interface) while the nonblue sand are static regions. The results in Fig. 6 were computed using an imaging frame rate of 1000 frames per second. Motion displacements were calculated relative to the image 20 frames prior (i.e. $0.02 \mathrm{~s}$ prior). The clustering shown is averaged over 100 clustering results.

The results in Fig. 6 indicate that the soil masses engaged by the rolling and static wheels are very different. The static wheel engages a much deeper soil mass, which is mainly behind the wheel center. On the other hand, the rolling wheel moves a relatively thin layer of soil from the front to the back of its ground contact. The depth of influence of the static wheel is estimated to be about three times that of the rolling wheel.

Fig. 7 reveals the relative velocities of the soil particles within the moving masses. Considering that the rim is not rotating, it's implied that the soil is simply pressed down and back without significant shear displacement at rim to soil interface. The soil appears to respond by grouping together and creating a deep unified mass that supports the wheel rim (the red region). At the point of failure, as imaged here (Fig. 7), the mass of soil finds its way to the soil surface. In contrast, the soil underneath the rolling wheel is guided by the path of the wheel rim. New soil from the lead edge of the wheel continuously enters the contact and is transported to the back of the wheel as it rotates. A relatively thin layer of soil moves at the same rate as the wheel rim. In addition, the velocity of the soil beneath the rim has a much more varied velocity, which depends on the circumferential position around the rim.

It is hypothesized that the soil structure formed by the static wheel is fundamentally stronger than that of the rolling wheel. Under the static load, the wheel is supported by a deeper and more thus more confined soil mass. The addition of rim motion, as in the case of the rotating wheel, undermines this support mechanism. Instead, the new soil is continuously brought into the contact region and the direction of soil motion is constantly changing as the soil moves around the rim. Strength is accumulated by shear displacement, but simultaneously lost by the change in shear direction. In addition any shear strength that is developed in the soil is ultimately lost when the soil exits contact.
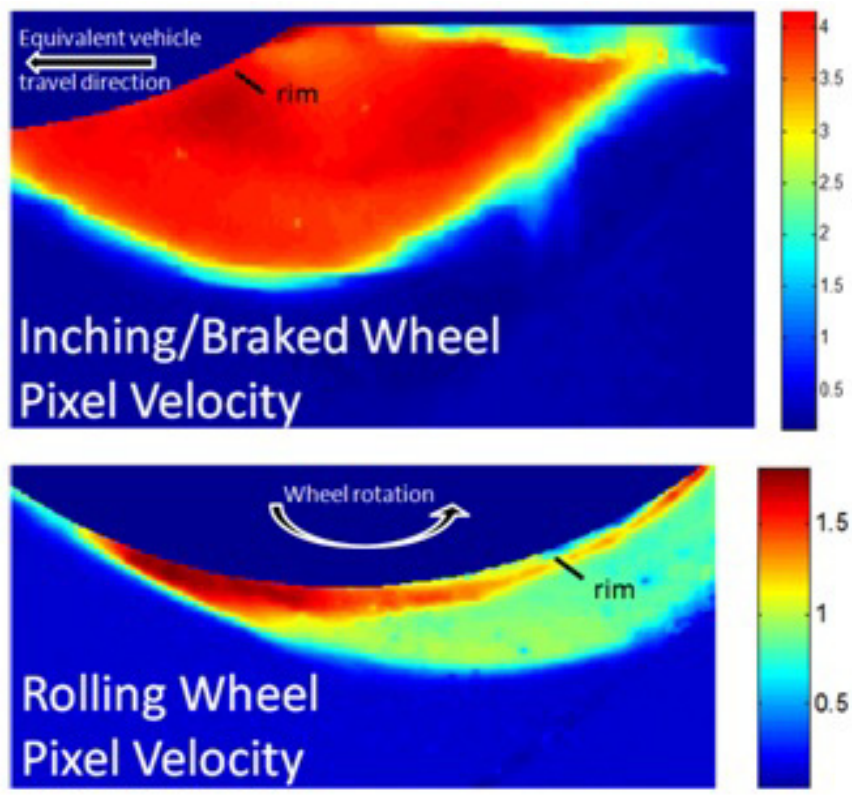

Figure 7. Soil particle velocity plot using optical flow analysis on inching and rolling wheel. The color map indicates the relative velocity of soil particles. Units of key are pixels/second velocity but flow geometry/direction is utilized in comparison instead of flow magnitude.

The flow or shear displacement (derived from Fig. 7) may be the key difference between the inching and rolling traction regimes. The flow of the simulant particles under the rigid wheel rim demonstrates that even at very low slip, a rigid rolling wheel induces high shear displacement due to the curvature of the wheel (many flat contact compliant wheels do not undergo this process). 
Specifically, soil particles must have a horizontal component of travel thus inducing high shear displacement which is expected to contribute the loss of strength along the shear interface. The static wheel does not force this process as there is little motion of the rim relative to the soil. It should be noted that a highly compliant wheel or track would have similar low shear displacement of the soil to that of the inching wheel.

The observed differences between the rolling and nonrolling wheel are generally not well represented by current analytical models and have been largely misleading when wheel performance design and analysis have been conducted in the past. Analytical models do not account for soil flow or slippage in their effect on shear strength and inadequate for use of wheel-soil interaction analysis [12]. This analysis of inching versus rolling could not have been undertaken without a primarily experimental approach.

\section{CONCLUSION}

This work demonstrated an implementation of the inching/push-roll locomotion technique on a full scale vehicle and conducted studies to investigate the source of the measured tractive gains (approximately double). Key differences of conventional rolling compared to inching were successfully portrayed, highlighting a unified deep soil mass created by a static wheel as a source that can react extremely high thrust. Further investigation into the loss of strength along the rolling interface could aid in design of high drawbar pull wheel designs as well as the efficient use of walking and inching locomotion. Ongoing experimentation investigating the shear displacement along the contact length of the wheel are currently being conducted. In addition, the continued use of the new experimental apparatus and image processing techniques developed during this study can aid in efforts to study wheel-soil interaction fundamentals and the design of wheels for space.

\section{REFERENCES}

[1] Wilcox, B.. "ATHLETE: A Cargo and Habitat Transporter for the Moon". IEEEAC. 2009.

[2] Harrison, D. "Next Generation Rover for Lunar Exploration." IEEEAC 2007.

[3] Patel, N. "The ExoMars rover locomotion subsystem." Journal of Terramechanics-, vol 47(4). pp.227-247. 2010

[4] D. Wettergreen, S. Moreland, K. Skonieczny, D. Jonak, D. Kohanbash, J. Teza, "Design and field experimentation of a prototype Lunar prospector," Int. J. Robotics Research, In press, 2010.

[5] von Sybel, H., \& Grosse-Scharmenn, F. "Increased Draft for Wheeled Vehicles Operating Outside the Roadway by the Thrust-Stride-System." Publication Source and Date Unknown.

[6] Zhongliang, H. "Study and Implementation of Wheel Walking for a Mars Rover." Masters Thesis . Space Science and Technology Department of Space Science, Kiruna. 2007.

[7] Bekker, M. "Theory of Land Locomotion," Ann Arbor: The University of Michigan Press, 1956.

[8] Czako, T. "An Analysis of Multi-Element Inching Vehicles." Technical report. Michigan: U.S. Army TankAutomotive Center, 1963.

[9] Wong, J. "Theory of Ground Vehicles." New York: Wiley Interscience. 2001.

[10] Oravec, H., Asnani, V., \& Zeng, X. "Design and characterization of GRC-1: A soil for lunar terramechanics testing in Earth-ambient conditions." Journal of Terramechanics, 47(6). pp. 361-377. 2010..

[11] Black, M. J. and Anandan, P. “The robust estimation of multiple motions: Parametric and piecewise-smooth flow fields," CVIU, 63(1), pp. 75-104. 1996.

[12] Wong, J. "Behaviour of Soil Beneath Rigid Wheels." Journal of Agricultural Engineering Research , vol 12(4) pp. 257-269. 1967.

[13] Bauer, R., Leung, W., Barfoot, T. "Development of a Dynamic Simulation Tool for the Exomars Rover," $8^{\text {th }}$ Int. Symposium on Artificial Intelligence, Robotics and Automation in Space, Munich. 2005. 


\section{BIOGRAPHY}

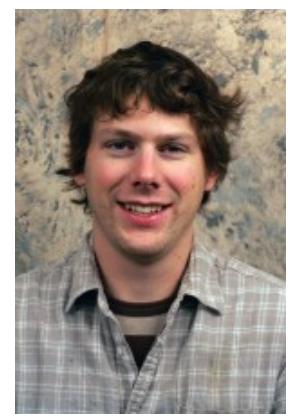

Scott Moreland is a Ph.D. student in the Carnegie Mellon University Mechanical Engineering Department. Scott completed his bachelor's degree in mechanical engineering at the University of Toronto and a masters degree in mechanical engineering at Carnegie Mellon in 2009. Currently, the core of his research at the Robotics Institute addresses understanding the mobility performance of vehicles in planetary environments such as the Moon or Mars for the application of space exploration.

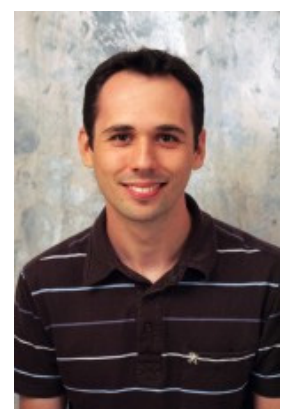

Krzysztof Skonieczny is a PhD candidate at the Carnegie Mellon University Robotics Institute, researching lunar mobility and excavation systems with the Field Robotics Center. He has Bachelor's and Master's degrees in aerospace engineering from the University of Toronto. He has worked with MDA Space Missions on operations and control for space robots including the Canadarm 2 (robotic arm for the International Space Station) and a prototype ExoMars rover (for the European Space Agency's flagship Mars mission). He also worked as an Operations Research analyst for the Canadian Department of National Defence, conducting field experiments of mobile ad hoc networks.

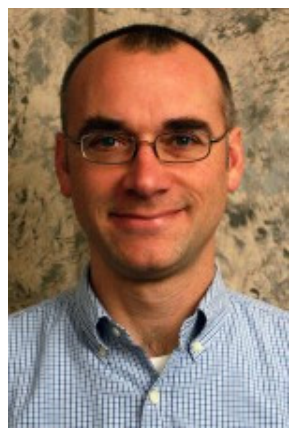

Dr. David. S. Wettergreen is an associate research professor at the Robotics Institute at Carnegie Mellon University. His research focuses on robotic exploration: underwater, on the surface, and in air and space, and in the necessary ingredients of perception, planning, learning and control for robot autonomy. His work spans conceptual and algorithm design through to field experimentation and results in mobile robots that explore the difficult, dangerous, and usually dirty places on Earth, in the service of scientific and technical investigations. Much of this work is relevant to ongoing and future space activities.

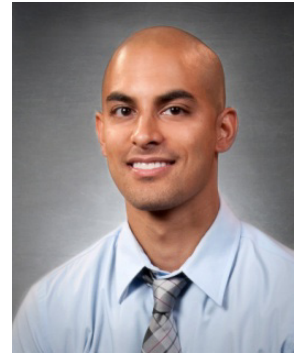

Vivake Asnani is a staff research engineer for NASA's Glenn Research Center in Cleveland, $\mathrm{OH}$. Vivake studied at the Ohio State University, earning a bachelor's degree in electrical engineering and a master's degree in mechanical engineering. Throughout his career, Vivake has worked on multidisciplinary technology development. For NASA's Space program, Vivake has worked with industry to develop non-pneumatic tires for use on planetary exploration and Earth vehicles. He has also worked extensively on evaluating the mobility of exploration vehicles, for terramechanics model development and to support vehicle design. For NASA's fundamental aeronautics program, Vivake develops technology to actively cancel vibration and sound generated by rotating machinery.

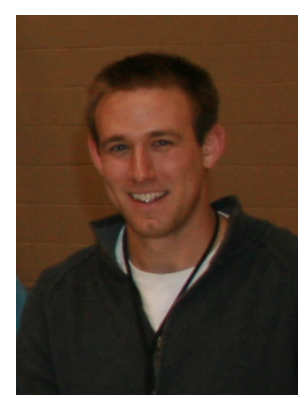

Colin Creager is a researcher at the NASA Glenn Research Center. He completed his Bachelor's degree in Mechanical Engineering at the Ohio State University in 2006. He is a member of the Surface Mobility Technology Team at GRC and conducts research on vehicle traction in soft terrain, focusing on Lunar and Planetary applications. His work targets ways to improve the mobility of robotic and manned vehicles on extra-terrestrial surfaces to enable more advanced space exploration.

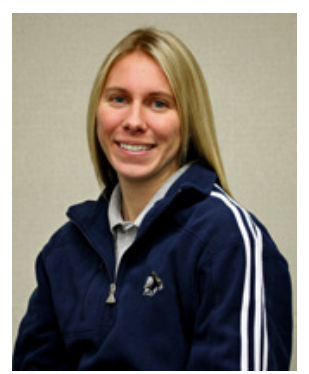

Dr. Heather Oravec is a Research Assistant Professor at the University of Akron's College of Engineering. Her current research focuses on the development of advanced aerospace seal technologies for the National Aeronautics and Space Administration's Low Impact Docking System relevant for future space missions. Her work covers a broad range of development activities from conceptual design, analytical modeling, experimental testing, and analysis of seals that will withstand the harshness of space and interplanetary missions. Other research interests include characterization of the lunar and Martian regolith for surface mobility operations and understanding the environmental effects on the mechanical properties of planetary regolith. 
